\title{
Dynamic characterization of an electrothermal actuator devoted to discrete MEMS positioning
}

\author{
I. Bouhadda, A. Mohand-Ousaid, P. Le Moal, G. Bourbon and P. Lutz, IEEE member. \\ Univ. Bourgogne Franche-Comté, Femto-st Institute, Besançon, France \\ Corresponding author: ismail.bouhadda@femto-st.fr
}

\begin{abstract}
This paper focuses on the dynamic characterization of an electrothermal actuator devoted to discrete MEMS positioning. Based on U-shape structure, such actuator has been employed in several MEMS applications where fine and repeatable positioning is required. The studied electrothermal actuator here is microfabricated on a doped SOI substrate and its dynamic response, during heating and cooling cycles, is recorded using precise and high-speed camera. To explain its dynamic behavior, FEM simulations, using Comsol multiphysics software facility, are carried out. The result of this numerical analysis shows a strong relationship between the temperature distribution and the displacement provided by the actuator. Finally, the influence of the dynamic behavior on the control of the actuator is discussed using experimental characterizations of its displacements under several voltage pulses with different frequencies.
\end{abstract}

Keywords- U-shaped actuator, MEMS, microfabrication, electrothermal actuator, thermomechanical.

\section{INTRODUCTION}

The U-shaped electrothermal actuator, operating on the principle of Joule heating and differential thermal expansion, is widely used in MEMS applications [1,2]. Such actuator offers many advantages: (i) large deflection with small output force around $10 \mathrm{mN}$ [3], (ii) small footprint, (iii) compliance with standard MEMS based fabrication process and so on. Combining these advantages makes the U-shaped electrothermal actuator suitable with a promising potential for micro/nanoscale applications such as micropositioning, microgripping and micromanipulation $[4,5]$.

In this paper we focus on the dynamic characterization of a U-shape electrothermal actuator devoted to discrete MEMS positioning application in particular digital microrobot. In this application, the digital robot employs a U-shape actuator in order to achieve micropositioning task. Acting on a bistable and two mechanical systems, the actuator allows to control two latches position: open and close, as described in [6,7]. In addition to static response, the actuator dynamic response is important in this case. In fact, an accurate characterization to control the actuator is highly required in order to achieve correctly the task. An unpredictable behavior of the actuator may damage the sample if the generated force by the actuator

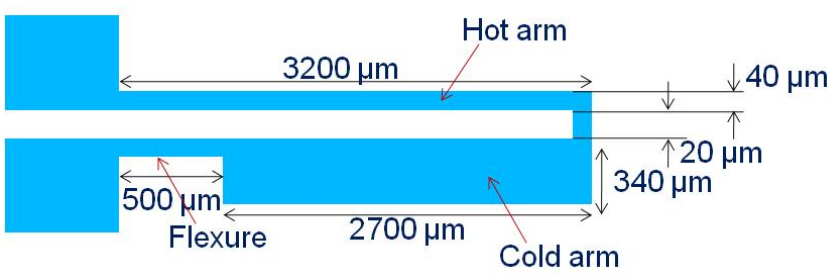

Fig.1. 2D schematic of U-shaped actuator.

exceeds the upper limit, or rather the opposite, inducing a poor performance of the actuator if the force is not high enough.

The first part of this paper deals with the device fabrication process, followed by experimental results obtained when different voltages are applied during heating and cooling cycles. The actuator behavior is then analyzed by using FEM simulations. In the second part, experimental characterizations of the displacement are carried out under several voltage pulses with different frequencies. Based on these experiments and FEM simulations results, the influence of the dynamic behavior on the actuator control is discussed. Finally, the work is summarized.

\section{ACTUATOR FABRICATION}

Figure 1 shows the U-shaped electrothermal actuator dimensions used in this study. Based on planar structure, the actuator is composed of three main parts: hot arm, cold arm and anchors. The basic principle of such actuator consists to exploit the thermal effect between its arms in order to provide a controlled deformation. In other words, the actuator deflects at its tip by unequal thermal expansion of the constituent arms [8].

The thermal actuators studied here were fabricated using $<100>$ oriented and highly doped p-type single-crystalline silicon-on-insulator (SOI) wafer, with resistivity of 0.01-0.03 ohm.cm. The SOI wafer is composed of $100 \mu \mathrm{m}$ thick device layer,a $2 \mu \mathrm{m}$ thick buried oxide (SiO2) and a 380thick $\mu \mathrm{m}$ handle layer with $2 \mu \mathrm{m}$ thick silicon dioxide layer which serves as a hard mask during the etching.

The backside oxide layer is patterned and etched in a buffered hydrofluoric acid (BHF) (Fig. 2-a). A layer of 200nm thick of gold is deposited and patterned on the device layer (Fig. 2-b). This later is lithographically patterned and etched using deep reactive ion etching DRIE process (Fig. 2-c). Then, the handle layer is also etched using DRIE process (Fig. 2-d). The last step of this process consists to release the useless parts from the wafer using HF wet etching as illustrated in Fig. 2-e. 
At the end of the process, the structure shown in Fig. 3 is obtained. This figure gives an optical capture of the microfabricated U-shape actuator.

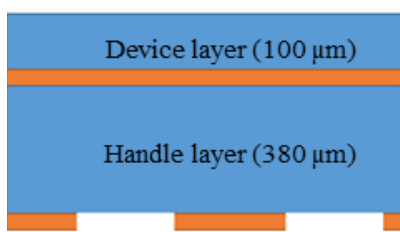

a) Wet etching of $\mathrm{SiO} 2$ (backside)

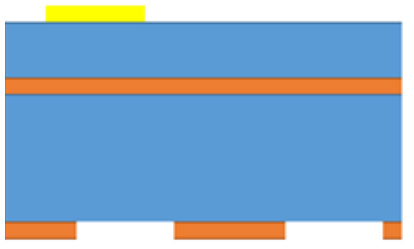

b) $\mathrm{Ti} / \mathrm{Au}$ deposition

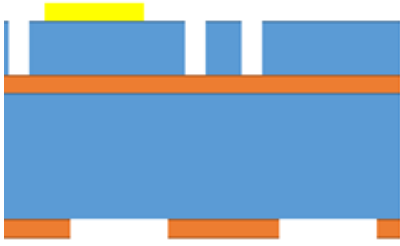

c) Dry etching of Si (frontside)

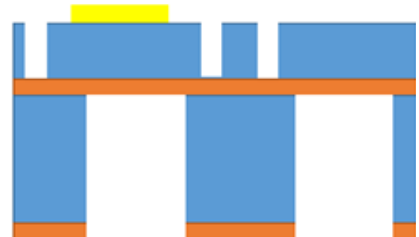

d) Dry etching of Si (backside)

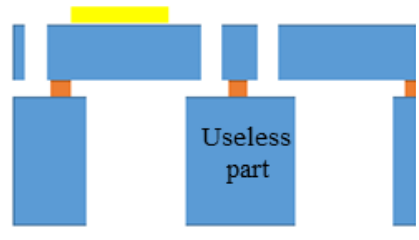

e) Wet etching of $\mathrm{SiO} 2$

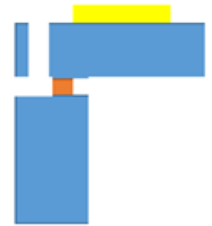

e) Useless part releasing

$\mathrm{SiO}_{2}(2 \mu \mathrm{m})$

Fig. 2. Fabrication process steps of the U-shaped actuator on SOI wafer.

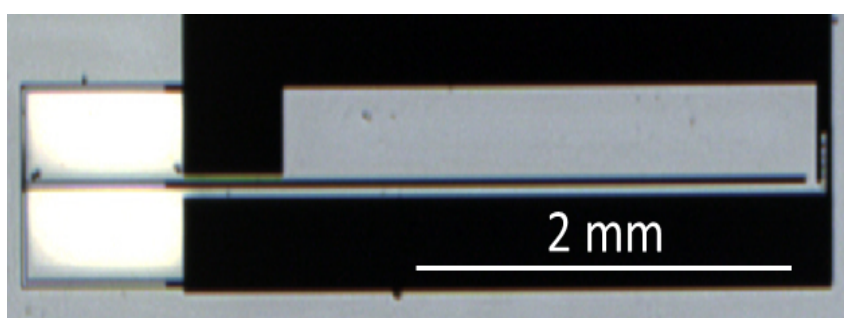

Fig. 3. Optical image of U-shaped actuator after fabrication.

\section{EXPERIMENTAL CHARACTERIZATION, RESULTS AND DISCUSSIONS}

The experimental set-up as depicted in Fig. 4 is composed of: a microfabricated actuator, a high-speed camera, amplifiers, an optical microscope and a desktop computer. The camera is used in order to detect the actuator displacement with a high rate frame record and then treated under pixel tracking software. These mechanical displacements are characterized under different square voltage signals, during heating (power on) and cooling cycles (power off).

Figure 5 shows the recorded actuator displacement during the square signal application, corresponding to a single heating and cooling cycle. The result shows here that during heating cycle, an overshoot of the actuator displacement is observed, followed by a steady state position. The displacement peak increases from 32 to $200 \mu \mathrm{m}$ when the applied voltage rises from 10 to $20 \mathrm{~V}$.

After power cut, during the cooling cycle, the actuator moves back to the negative side and returns slowly to the rest position. In the same way as the heating cycle, here the displacement peak increases from -11 to $-51 \mu \mathrm{m}$ when the applied voltage rises from 10 to $20 \mathrm{~V}$.

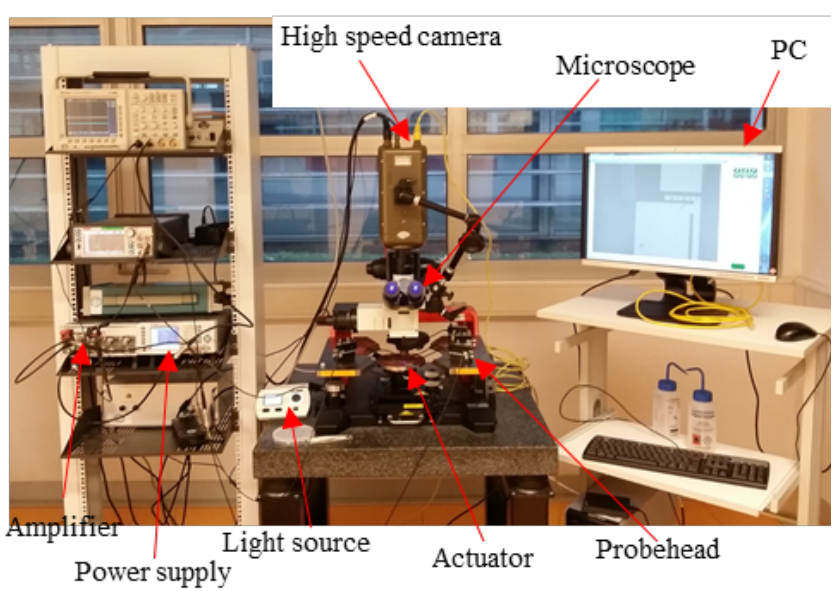

Fig. 4. Experimental setup.

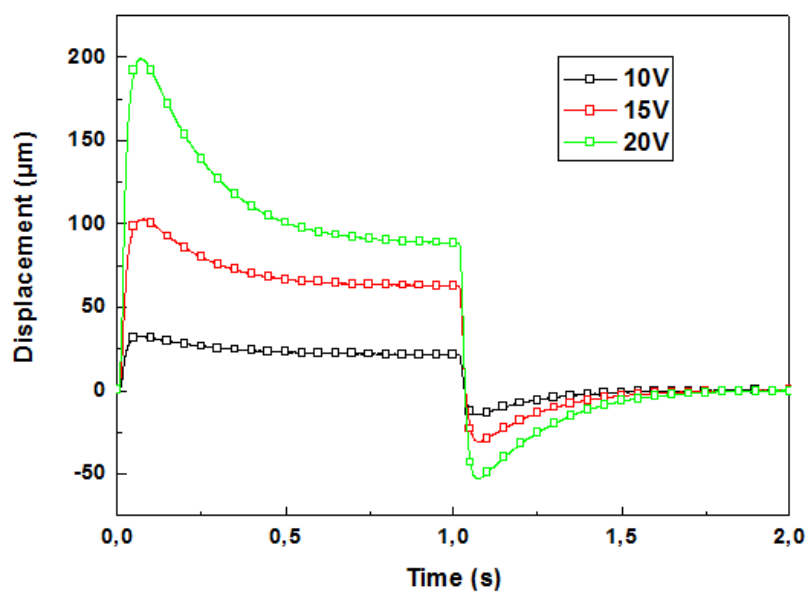

Fig. 5. Displacement curves of the U-shaped actuator according to time for different voltage amplitudes.

For a better understanding of the actuator behavior under voltage application, FEM simulations are conducted using Comsol multiphysics. These simulations are useful to highlight the temperature distribution effect, which is difficult to assess experimentally. The used module is Joule heating multiphysics in which the electrical and thermal properties are coupled. The electrical and thermal conductivity are thermally dependent. These parameters are defined by using the following equations:

$$
\begin{gathered}
\alpha=10^{-6} \cdot\left(3.725\left(1-e^{-5.88 \cdot 10^{-3}(T-124)}\right)+5.548 \cdot 10^{-4} T\right. \\
\sigma=\frac{1}{\rho_{0}\left(1+\alpha\left(T-T_{S}\right)\right)}
\end{gathered}
$$

Where $T_{s}$ is the initial temperature and $\rho_{0}$ is the resistivity at $\mathrm{T}_{\mathrm{s}}$. Other physical constants are listed in table 1 .

\begin{tabular}{ll}
\hline Parameter & Value \\
\hline Young' modulus & $\mathrm{E}=169 \cdot 10^{9} \mathrm{~Pa}$ \\
Thermal expansion & $\alpha=2.6 \cdot 10^{-6} \mathrm{~K}^{-1}$ \\
Thermal conductivity & $\mathrm{k}=149 \mathrm{~W} /(\mathrm{m} \cdot \mathrm{K})$ \\
Substrate temperature & $\mathrm{Ts}=293 \mathrm{~K}$ \\
Electrical resistivity at $\mathrm{T}_{\mathrm{s}}$ & $\rho_{0}=2.6 \cdot 10^{-4} \Omega \cdot \mathrm{m}$ \\
\hline Table 1. Parameters used in this analysis.
\end{tabular}

Fig. 6 shows the resulted steady state temperature profile along hot/cold arm when a voltage of amplitude $15 \mathrm{~V}$ is applied. Due to the lower width, the local Joule heating is higher in the hot arm. However, despite the lower width of the 
flexure, the temperature profile is limited by the cold temperature of the anchor and the cold arm at the steady state.

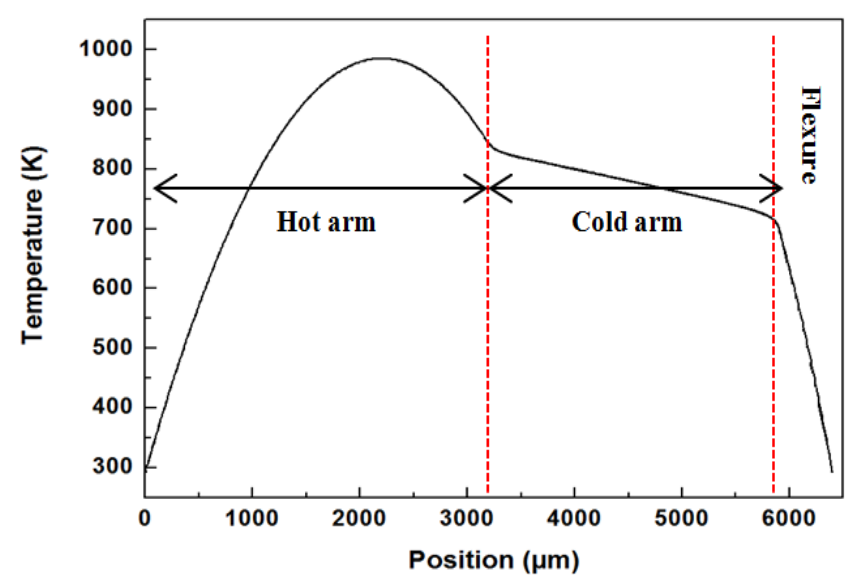

Fig. 6. Steady state temperature profile $\mathrm{T}(\mathrm{x})$ along the hot/cold arm thermal actuator at $15 \mathrm{~V}$.

Figure 7 shows the mean temperature evolution according to time of both hot and cold arms, and flexure after $15 \mathrm{~V}$ voltage application.

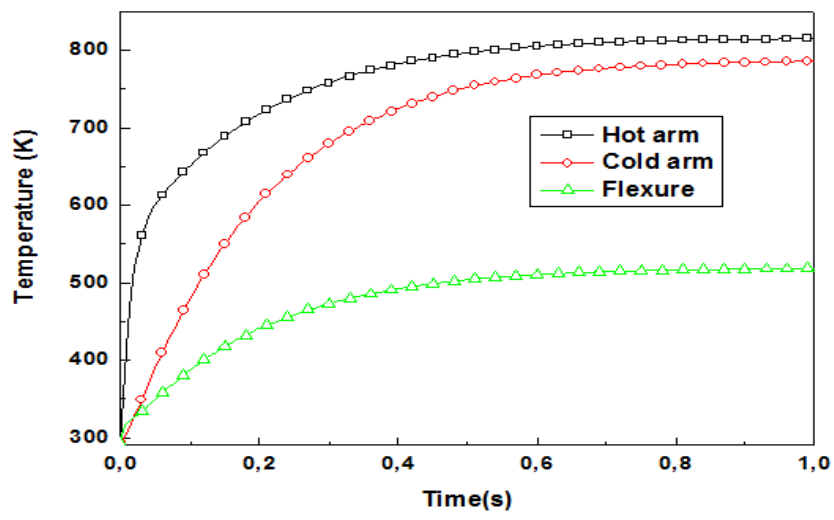

Fig. 7. Evolution of the mean temperature according to time in the three arms of the actuator when applying a voltage of $15 \mathrm{~V}$.

According to this result, the temperature distribution in the hot arm rapidly reaches the steady state comparing to the other arms. The response time difference of each arm explains the displacement overshoot observed previously (see Figure 5). The steady state of displacement is obtained when the max temperature in the cold arm is reached.

The linear thermal expansion for both arms and flexure can be calculated using the following equations:

$$
\begin{gathered}
\Delta L_{c}=\alpha \int_{0}^{L_{c}}\left(T_{c}(x)-T_{s}\right) d x=\alpha L_{c}\left(\overline{T_{c}}-T_{s}\right) \\
\Delta L_{c}=\alpha \int_{0}^{L_{h}}\left(T_{h}(x)-T_{s}\right) d x=\alpha L_{h}\left(\overline{T_{h}}-T_{s}\right) \\
\Delta L_{h}=\alpha \int_{0}^{L_{f}}\left(T_{f}(x)-T_{s}\right) d x=\alpha L_{f}\left(\overline{T_{f}}-T_{s}\right)
\end{gathered}
$$

Where $\bar{T}_{c}, \overline{T_{h}}, \bar{T}_{c}$ represent the average temperature of the hot arm, cold arm and flexure, respectively.

The expansion values are calculated for each arm using equations (3), (4) and (5). Their time evolution is reported in Figure 4.



Fig.8. Thermal expansion of the hot, cold and flexure arms, cold side and expansion difference between both sides of the actuator after applying a voltage of $15 \mathrm{~V}$.

As shown in figure 8, the thermal expansion in the hot arm is larger than the cold arm expansion. This difference is due to the high length and the high temperature induced in the hot arm, responsible of such larger expansion compared to the cold arm. It is important to notice here that the expansion difference between the three different arms $\left(\Delta \mathrm{L}_{\mathrm{h}}-\Delta \mathrm{L}_{\mathrm{c}}-\Delta \mathrm{L}_{\mathrm{f}}\right)$ follows the same form of displacement curves.

As result, the displacement of the actuator follows the temperature difference, between the hot arm and the cold side (cold arm + flexure), with an overshoot followed by a stabilization of the position.

The negative displacement during the cooling cycle is also related to the temperature distribution variation within the actuator. Due to its consequent volume, the cold arm stores larger heat energy during the heating cycle compared to the other arms. As a consequence, the hot arm requires shorter time to evacuate the cumulated heat compared to the cold arm, generating a new temperature difference between the two arms. This new temperature gradient is responsible of the displacement in the negative side. Finally the actuator moves back to the rest position until the cold arm evacuates its cumulated heat.

For a proper use, the actuator control is highly important and has to be considered. Commonly, one can benefit from the actuator performance (force, displacement) before this later reaches its displacement peak. Thus the provided power supply duration has to be taken into account in order to promote the actuator use. In order to optimize this duration, here we performed displacement measurements under 12 applied voltage pulses of $15 \mathrm{~V}$ magnitude and two different frequencies of $10 \mathrm{~Hz}$ and $50 \mathrm{~Hz}$, figure 9 .

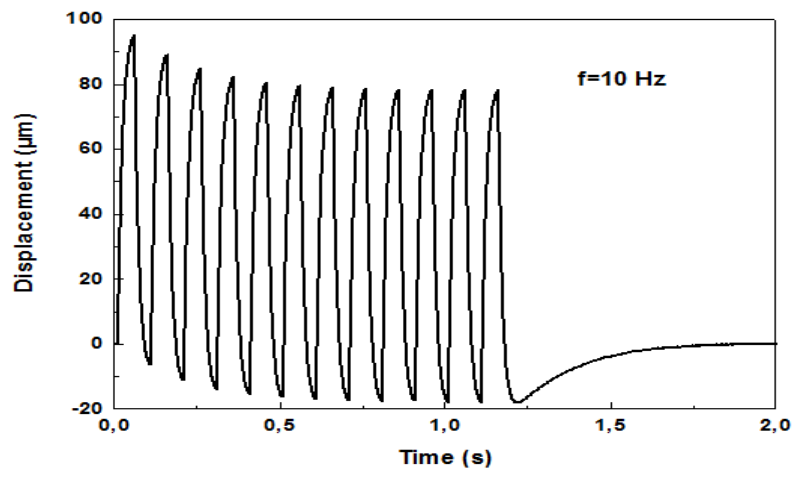




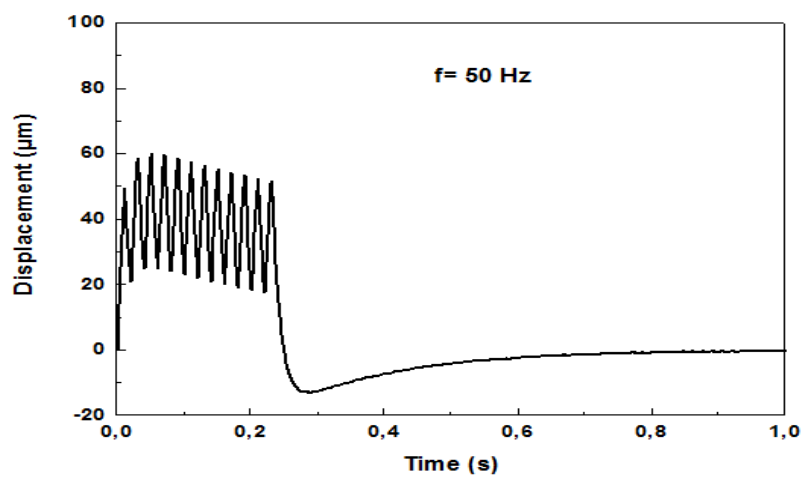

Fig. 9. Displacement curves of the actuator after applying 12 potential pulses at 10 and $50 \mathrm{~Hz}$.

For a signal frequency of $10 \mathrm{~Hz}$, we observe a decrease of displacement magnitude after the first voltage pulse. The displacement stabilization is observed after 5 consecutive pulses.

This behavior can be explained by the fact that the hot arm rapidly reaches the max temperature during the heating cycle, while the temperature of the cold arm gradually increases until it reaches the upper value. When the cold arm reaches the maximum temperature, the stabilization can be observed from the fifth pulse here (Fig.9.a). The temperature rising in the cold arm also generates the displacement increase in the negative side during the cooling cycles, until the maximum displacement magnitude is reached after the fifth pulse.

For $50 \mathrm{~Hz}$, the peak displacement increases until the third pulse (called phase one), then decreases. This behavior indicates that during the increasing phase, the hot arm has not reached her temperature steady state yet. Besides in the second phase the peak displacement decreases because of the risen temperature in the cold arm, whereas the hot arm is already in the steady state.

Note that the displacement peaks at $50 \mathrm{~Hz}$ are smaller than obtained ones at $10 \mathrm{~Hz}$. The displacement at $50 \mathrm{~Hz}$ in the cooling cycles does not return to the original position. This can be explained the high frequency, indeed the actuator heating and cooling time are not fast enough for such high frequency. This highlights the importance of the signal frequency, for a better actuator control, the frequency has to be tuned according to the cooling time.

\section{CONCLUSION}

U-shaped electrothermal actuator is fabricated on SOI substrate and its displacement is recorded using high-speed camera after applying voltage pulses. The actuator behavior during the voltage application is explained by numerical simulations. Indeed, the actuator displacement during the heating cycle follows the temperature difference, between the hot arm and the cold side (cold arm+ flexure). An overshoot is obtained followed by position stabilization. The influence of the dynamic behavior on the actuator control is discussed by performing displacements measurements with 12 applied voltage pulses of $15 \mathrm{~V}$ at 10 and $50 \mathrm{~Hz}$.

\section{ACKNOWLEDGMENT}

This work has been supported by the Labex ACTION project (contract ANR-11-LABX-01-01), the French RENATECH network and its FEMTO-ST technological facility.

\section{REFERENCES}

[1] S.-C. Chen and M. L. Culpepper, "Design of contoured microscale thermomechanical actuators," Journal of Microelectromechanical Systems, vol. 15, no. 5, pp. 1226-1234, 2006.

[2] Z. Wang, X. Shen, and X. Chen, "Design, modeling, and characterization of a mems electrothermal microgripper," Microsystem Technologies, pp. 1-8, 2015.

[3] B. Sahu, C. R. Taylor, and K. K. Leang, "Emerging challenges of microactuatorsfor nanoscale positioning, assembly, and manipulation,"Journal of manufacturing science and engineering, vol. 132, no. 3, p.030917, 2010.

[4] Q. Chen, Y. Haddab and P. Lutz, "Microfabricated Bistable Module for Digital Microrobotics", in Journal of Micro-Nano Mechatronics, pringerBerlin / Heidelberg, vol. 6, pp. 1-12, 2010.

[5] V Chalvet, Y Haddab, P Lutz, "A microfabricated planar digital microrobot for precise positioning based on bistable modules" IEEE Transactions on Robotics 29 (3), 641-649, 2013.

[6] H. Hussein , G. Bourbon, P. Le Moal, Y. Haddab, P. Lutz "Mechanical stop mechanism for overcoming MEMS fabrication tolerances" Journal of Micromechanics and Microengineering 27(1):017001, 2017

[7] H. Hussein "Contribution to Digital Microrobotics: Modeling, Design and Fabrication of Curved Beams, U-shaped Actuators and Multistable Microrobots" Ph.D. Thesis, UBFC university, 2015.

[8] J. H. Comtois, M. A. Michalicek and C. C. Barron, "Characterization of electrothermal actuators and arrays fabricated in a four-level, planarized surface-micromachined polycrystalline silicon process," Solid State Sensors and Actuators, 1997. TRANSDUCERS '97 Chicago., 1997 International Conference on, Chicago, IL, 1997, pp. 769-772 vol.2. doi: 10.1109/SENSOR.1997.635213

Ismail Bouhadda received a Master of physics of semiconductors and dielectrics in 2010 from the University of USTHB in Algeria and Ph.D in electronic from the University of Rennes1 in 2014. His research interest includes the fabrication of chemical, biological or mechanical sensors using bulk or surface micromachining, microfluidic devices, organic/inorganic thin film transistors and MEMS. 